\title{
Qualidade de Vida e Valores nas Organizações: Impactos na Confiança do Empregado
}

\author{
Uanisléia Lima da Silva \\ Universidade Federal de Uberlândia, MG, Brasil.
}

\author{
Áurea de Fátima Oliveira \\ Universidade Federal de Uberlândia, MG, Brasil.
}

Resumo: Os estudos sobre as relações empregado-organização são favorecidos pela compreensão de que recursos humanos são parte fundamental do processo organizacional. A confiança é um aspecto importante nesse processo, podendo influenciar a eficácia e o desempenho organizacionais. Assim, este estudo teve por objetivo verificar o poder de predição da percepção de valores organizacionais e da qualidade de vida no trabalho (QVT) sobre a confiança do empregado na organização. A amostra foi composta por 188 indivíduos formalmente empregados que responderam instrumentos fidedignos e válidos relativos aos construtos investigados. Os dados foram analisados mediante estatística descritiva e análises de regressão múltipla padrão. Os resultados mostraram que as escalas apresentaram índices de confiabilidade adequados para a amostra do estudo. As análises de regressão indicaram que os valores organizacionais e fatores de QVT foram preditores significativos de componentes éticos e competência organizacional. Oportunismo foi explicado somente pelos valores domínio, prestígio e coletividade. Nota-se que os valores, assim como os fatores de QVT, contribuíram de forma diferenciada na explicação das variáveis critério. Os resultados sugerem a necessidade de ampliar o modelo de investigação dos antecedentes da confiança do empregado na organização visando a melhor compreender a formação deste vínculo.

Palavras-chave: Confiança, Qualidade de Vida no Trabalho, Valores, Cultura.

\section{Quality of Life and Values in Organizations: Impacts on the Employee Trust}

\begin{abstract}
The studies of employee-organization relationships are favored by the understanding that human resources are a key part of the organizational process. Trust is an important aspect in this process and may influence the effectiveness and organizational performance. Thus, this study aimed to verify the predictive power of the perceived organizational values and quality of work life (QWL) on the employee trust in the organization. The sample consisted of 188 formally employed individuals who responded reliable and valid instruments for the constructs investigated. Data were analyzed using descriptive statistics, and analysis of standard multiple regression. The results showed that the scales presented suitable reliability indices for the study sample. Regression analyses indicated that organizational values and QWL factors were significant predictors of organizational competence and ethical components. Opportunism was explained only by domain, prestige and collective values. It was observed that the values, as well as factors of QWL, contributed differently in explaining the criterion variables. The results suggest the need to expand the antecedents' research model of the employee's trust in the organization to better understand the formation of this bond.
\end{abstract}

Keywords: Trust, Quality of Work Life, Values, Culture. 


\title{
Calidad de Vida y Valores en las Organizaciones: Impactos en la Confianza del Empleado
}

\begin{abstract}
Resumen: Los estudios acerca de las relaciones empleado-organización son beneficiados por la comprensión de que los recursos humanos son parte fundamental del proceso organizacional. La confianza es un elemento importante en este proceso, pudiendo influir en la eficiencia y en el desempeño organizacional. Por consiguiente, este trabajo tiene como objetivo averiguar el poder de predicción de la percepción de valores organizacionales y de la calidad de vida en el trabajo (CVT) sobre la confianza del empleado en la organización. La muestra fue formada por 188 sujetos empleados que respondieron instrumentos fidedignos y válidos con relación a lo que investigamos. Los datos fueron analizados por medio de estadística descriptiva y análisis de regresión múltiple patrón. Los resultados demostraron que las escalas presentaron índices de confiabilidad adecuados para la muestra del estudio. Los análisis de regresión indicaron que los valores organizacionales y factores de CVT fueron precursores significativos de componentes éticos y de competencia organizacional. Oportunismo fue explicado solamente por los valores de dominio, prestigio y colectividad. Se puede percibir que los valores, así como los factores de CVT, contribuyeron de manera diferenciada en la explicación de las variables criterio. Los resultados sugieren la necesidad de ampliar el modelo de investigación de los precedentes de la confianza del empleado en la organización, buscando comprender mejor la formación de este vínculo.
\end{abstract}

Palabras clave: Confianza, Calidad de Vida en el Trabajo, Valores, Cultura.

\section{Introdução}

As organizações impulsionadas pelas transformações sociais, políticas e econômicas tendem a compreender que a forma mercadológica mais competitiva é a valorização dos recursos humanos, pois a força humana é a unidade base de trabalho para se atingir a produção e o desenvolvimento almejados (Sinha, 2012; Valizadeh, \& Ghahremani, 2012). Gorenak e Košir (2012) salientam que nem mesmo o melhor equipamento é capaz de garantir o sucesso de organizações caso estas não tenham pessoas envolvidas em fazer o trabalho necessário, o que favorece os estudos sobre as relações empregado-organização.

$\mathrm{O}$ incentivo às novas estratégias de gestão de negócios, motivado pelas mudanças ocorridas no contexto organizacional, faz surgir ferramentas que visam a fortalecer o desempenho das organizações (Ianaguivara, 2011). Nesse contexto, a confiança, além de ser considerada uma parte essencial da eficácia e do desempenho organizacionais (Guardani, Teixeira, Bido, \& Mazzon, 2013; Purohit, Patel, \& Purohit, 2014; Van der Berg \& Martins, 2013), é uma das bases mais importantes da interação social e, portanto, dos relacionamentos interpessoais (Oliveira, \& Souza, 2014; Oliveira,
\& Tamayo, 2008) e do relacionamento do empregado com a organização em que se trabalha.

A confiança do empregado na organização é um construto multidimensional de base cognitiva, ou seja, é o conjunto de crenças do empregado a respeito de componentes éticos da organização, competência organizacional e uso limitado de oportunismo em suas relações (Ianaguivara, 2011, p. 81). Diversos benefícios consequentes da confiança organizacional são suficientes para justificar a necessidade de se conhecer os aspectos que propiciam o seu surgimento (Batista, \& Oliveira, 2012) e incluem: favorecimento do trabalho em equipe, liderança, definição de metas e avaliação de desempenho (Watson, 2005), melhoria da interação empregado-organização, diminuição de conflitos e dificuldades na comunicação (Fard, Zahed-Babelan, \& Sattari, 2013), bem como menor necessidade de controle e maior estabilidade e cooperação espontânea nas relações (Batista, \& Oliveira, 2012; Kramer, 1999). Assim, é possível que a confiança organizacional permita uma relação mais tranquila e harmoniosa dos envolvidos e que haja fatores organizacionais que favoreçam a experiência de confiar (DeConinck, 2010; Kramer, 1999; Oliveira, 2004; Van der Berg, 2011). 
As organizações e os estudiosos reconhecem que os recursos humanos são o principal patrimônio de organizações bem-sucedidas, e por isso, investem cada vez mais em treinamento e qualidade de vida no trabalho, já que para se inserir de forma vantajosa no mundo globalizado, faz-se necessário manter funcionários saudáveis, motivados e qualificados (Siqueira, \& Kurcgant, 2012, p. 152). Nesse sentido, é pertinente abordar que o conceito de saúde determinado pela Organização Mundial de Saúde (OMS) está além do que se refere à ausência de doença, ou seja, não se trata apenas da abordagem patológica, mas das condições biopsicossociais do indivíduo. As diversas definições de determinantes sociais de saúde expressam o conceito de que as condições de vida e de trabalho dos indivíduos estão diretamente relacionadas com sua situação de saúde (Buss, \& Pellegrini Filho, 2007).

Os estudos acerca da Qualidade de Vida no Trabalho (QVT) originaram-se na década de $1950 \mathrm{com}$ a reformulação da abordagem sociotécnica para a organização do trabalho, contemplando a satisfação e o bem-estar do trabalhador (Tolfo, \& Piccinini, 2011). Após sofrer um declínio de interesse a partir de 1974, a QVT voltou a ser motivo de interesse nas décadas de 1980-1990, motivada, principalmente, pelo sucesso econômico japonês, que despertou o interesse dos pesquisadores a investigarem suas técnicas gerenciais voltadas para o aumento da competitividade internacional (Medeiros, 2007; Sinha, 2012).

A QVT é constituída a partir dos aspectos econômicos, psicológicos, organizacionais e sociais do trabalho (Khetavath, 2013), possibilitando a mensuração do impacto que o trabalho oferece sobre o grau de bem-estar do indivíduo em relação a aspectos diversos desse trabalho (Hackman, \& Oldham, 1975). Silva e Tolfo (1999) e Tolfo, Silva e Luna (2009) consideram que a QVT é uma manifestação objetiva da cultura organizacional. Geralmente, o estudo da cultura organizacional pressupõe o estudo do conjunto dos valores organizacionais (Oliveira, \& Tamayo, 2004) e faz-se relevante para a compreensão do comportamento nas organizações, pois assume o papel de legitimadora de tais valores. Os valores organizacionais, ao serem partilhados, atuam como elementos integradores e orientadores, influenciando as interações sociais e o modo como os membros de uma organização pensam, sentem e agem (Estivalete, Andrade, Gomes, \& Costa, 2012; Pires, 2004; Silva, \& Zanelli, 2004; Silva, Zanelli $\&$ Tolfo, 2014). Os valores organizacionais constituem uma dimensão fundamental da cultura organizacional, expressando as opções feitas pela organização através de sua história, padrões de comportamentos, de tomada de decisão, estruturas organizacionais e estratégias de gerenciamento de pessoas e ambientes (Gorenak, \& Košir, 2012; Pinto Neto, 2011; Tamayo, 1998). No contexto organizacional ocorrem interações entre estas variáveis, sendo que a confiança tem sido estudada como consequência de atributos e características das organizações, bem como dos arranjos e estruturas organizacionais (Rousseau, Sitkin, Burt, \& Camerer, 1998; Van der Berg, 2011).

Neste estudo, a confiança será abordada como variável dependente, supondo que quanto mais a organização tenha valores percebidos e compartilhados por seus funcionários, assim como adote medidas para melhor QVT, tanto mais haverá confiança na organização, o que poderá favorecer o comprometimento e a produtividade do empregado.

As literaturas internacional e nacional mostram diversos estudos que abrangem as variáveis propostas neste estudo (An, Yom, \& Ruggiero, 2011; Bigliardi, Dormio, Galati, \& Schiuma, 2012; Estivalete \& Andrade, 2012; Fard et al., 2013; Gorenak, \& Košir, 2012; Gorzoni, 2010; Medeiros, \& Ferreira, 2011; Oliveira, \& Tamayo, 2008; Özyilmaz, 2010), porém o modelo de investigação sugerido visa suprir uma lacuna nos estudos que abrangem tais construtos, o que acredita-se representar uma contribuição acadêmica importante no âmbito da Psicologia Organizacional e do Trabalho. Na perspectiva do gerenciamento de recursos humanos, os resultados deste estudo possibilitam a reflexão de aspectos que podem tornar a experiência do trabalho mais saudável e gratificante para o trabalhador.

\section{Método}

\section{Participantes}

Foi utilizada uma amostra de conveniência composta por 188 trabalhadores, cujo tamanho atende ao cálculo do poder do teste, considerando o efeito médio ( $\left.\mathrm{f}^{2}=0,15 ; \mathrm{p}<0,05\right)$, que indicou o número igual a 184 casos. A maioria dos participantes do estudo $(58,55 \%$; 110) é do gênero feminino, com idade média de 30,1 anos (DP $=9,3)$. O grau de instrução se concentrou acima do ensino médio, sendo que entre superior incompleto $(34,0 \%$; 64$)$ e pós-graduação $(28,7 \%$; 54$)$ totalizaram-se 
82,4\% da amostra (155 casos). O tempo de trabalho na organização atual, assim como o tempo de trabalho no cargo ocupado variaram entre 3 meses e 35 anos, com médias de 4,63 anos ( $\mathrm{DP}=6,81$ ) e 3,11 anos ( $\mathrm{DP}=4,54)$, respectivamente, sendo que a maioria dos trabalhadores estava vinculada a empresas privadas $(63,8 \% ; 120)$ e da área de prestação de serviços (83,5\%; 157).

\section{Instrumentos}

Foram utilizados os seguintes instrumentos:

a) Escala de Indicadores de Satisfações Contextuais - EISC (David, 2005) composta por 27 itens distribuídos em quatro fatores: Relações interpessoais ( 7 itens e Alpha $=0,90$ ); Segurança (12 itens; Alpha = 0,85); Ambiente físico adequado e seguro (5 itens e Alpha $=0,72$ ) e Saúde física (3 itens e Alpha $=0,71$ ). A escala de respostas é de sete pontos (tipo Likert), variando de 1 (fortemente insatisfeito) a 7 (fortemente satisfeito);

b) Inventário de Perfis de Valores Organizacionais (Oliveira, \& Tamayo, 2004) composto de 48 itens que englobam oito fatores: Realização, Conformidade, Domínio, Tradição, Prestígio, Autonomia, Coletividade e Bem-Estar do Empregado. A escala de resposta é constituída por seis pontos, variando de 1 (Não se parece em nada com minha organização) a 6 (É muito parecida com minha organização). A precisão do instrumento (Alpha de Cronbach) variou de 0,75 a 0,87;

c) Inventário de Confiança do Empregado na Organização (Ianaguivara, 2011) apresenta três fatores: Componentes Éticos, Competência Organizacional e Oportunismo, com Alphas de Cronbach de 0,84 a 0,96 . As respostas aos 42 itens são apresentadas na escala de cinco pontos $(1=$ discordo totalmente até $5=$ concordo totalmente). Por fim, os seguintes dados pessoais e funcionais dos participantes foram coletados: idade, gênero, grau de instrução, tempo de trabalho na organização, tipo de organização (privada ou pública) e tipo de atividades (comércio, indústria, serviços).

\section{Procedimentos de coleta e análise de dados}

A pesquisa foi aprovada pelo Comitê de Ética e Pesquisa da Universidade Federal de Uberlândia, conforme as normas da Resolução no 466/12 do Conselho
Nacional de Saúde - CNS para realização de pesquisa envolvendo seres humanos, com Parecer no 502.838.

Para análise dos dados, utilizou-se o programa estatístico SPSS (Statistical Package of Social Science, versão 20). Na análise exploratória dos dados foi verificada a precisão do arquivo de dados e as respostas omissas por serem aleatórias e inferiores a $5 \%$ foram substituídas pela média da amostra (Tabachnick, \& Fidell, 2001).

Os casos extremos univariados foram mantidos no banco de dados por serem moderados e os sete casos extremos multivariados foram eliminados, de forma que a amostra passou a conter 181 participantes. Diferenças de assimetria foram evidenciadas na análise dos histogramas com superposição da curva normal e dos índices de assimetria, permanecendo dentro dos parâmetros descritos por Miles e Shevlin (2001) que consideram que, ainda que os dados da amostra não apresentem uma distribuição normal, é considerado aceitável um índice de assimetria de até 2,0. Além disso, Tabachnick, e Fidell (2001) também afirmam que análise de regressão é uma técnica robusta à violação de pressupostos.

A multicolinearidade e singularidade não afetam os dados, pois as correlações bivariadas entre as variáveis do estudo permaneceram com $\mathrm{r}<0,90$, (Tabachnik, \& Fidell, 2001). Além disso, os valores de fatores de inflação da variância (VIF) estão todos abaixo de 10 e todas as tolerâncias acima de 0,20, confirmando que a colinearidade não afeta o conjunto dos dados (Field, 2009).

As estatísticas descritivas (média, frequência e desvio padrão) foram utilizadas para descrever a amostra e verificar os níveis de QVT, percepção de valores organizacionais e confiança do empregado na organização referente à amostra enquanto as correlações entre as variáveis foram verificadas por meio da correlação de Pearson. A confiabilidade dos instrumentos para a amostra do estudo foi verificada por meio do cálculo do Alpha de Cronbach, que revelou resultados aceitáveis (Hair, Anderson, Tatham, \& Black, 2005) e próximos aos valores originais, variando de 0,70 a 0,97 .

Por fim, foram calculados três modelos de regressão múltipla padrão, tendo como variáveis independentes os quatro fatores de QVT e os oito fatores de percepção de valores organizacionais e como variáveis critério as três dimensões da confiança do empregado na organização (Componentes Éticos, Competência e Oportunismo). 


\section{Resultados e discussão}

\section{Análises descritivas}

O cálculo das médias e desvios padrão de cada variável do estudo (Tabela 1) demonstrou que os trabalhadores participantes da pesquisa apresentaram níveis moderados de percepção dos valores organizacionais, de QVT e de confiança na organização. Para a análise da intensidade das correlações foram adotados os critérios propostos por Dancey e Reidy (2006), cujas correlações até 0,39 são consideradas fracas, de 0,40 a 0,69 , são moderadas e acima desses valores são consideradas fortes.

A Tabela 1 indica que houve correlações entre todas as variáveis, sendo a maioria significativa, com coeficientes considerados de fracos $(r=-0,15)$ a fortes $(r=0,82)$. As correções entre as variáveis critério (Confiança na Ética, na Competência e Oportunismo) e as variáveis explicativas foram, em sua maioria, moderadas e significativas, exceto no caso do fator de QVT Saúde Física, cuja correlação com confiança na competência organizacional não foi significativa. Resultado este que difere daquele encontrado por Batista e Oliveira (2012) no qual a relação indica que o empregado associa o cuidado da organização com seu bem-estar, sua satisfação e possibilidade de solução de problemas, com a crença de que a organização possui solidez e, portanto, é confiável.

\section{Análises de regressão múltipla}

Os resultados da primeira análise de regressão (Tabela 2) mostram que os preditores significativos de confiança na ética da organização foram os valores organizacionais Bem-Estar do Empregado $(\beta=0,26$, $\mathrm{t}=4,31, \mathrm{p}<0,001)$, Coletividade $(\beta=0,43, \mathrm{t}=7,13$, $\mathrm{p}<0,001)$ e os fatores de QVT Relações Interpessoais ( $\beta=0,25, \mathrm{t}=4,47, \mathrm{p}<0,001)$ e Segurança $(\beta=0,14$, $\mathrm{t}=2,22, \mathrm{p}<0,028)$. A variância explicada neste modelo é de $82 \%\left(\mathrm{R}^{2}\right.$ ajustado $=0,81 ; \mathrm{F}(12,168)=63,05$; $\mathrm{p}<0,001)$. Os resultados indicam que a percepção de valores coletivistas de base ética, foco na satisfação do empregado e qualidade das relações com chefes e colegas assim como a qualidade da oferta de salários e benefícios ao trabalhador são aspectos que propiciam uma relação de confiança pautada na honestidade, respeito e transparência.

Tzafrir e Dolan (2004) alegam que vários teóricos têm mostrado o papel desempenhado pelos valores par- tilhados quanto ao estabelecimento de elevados níveis de confiança, assim como Paula (2009), que afirma ser a confiança do empregado na organização normativa, podendo se basear em suas normas e em seus valores. Os resultados possibilitam traçar um paralelo com o trabalho de Fard et al. (2013) que, ao estudarem a relação entre confiança e cultura organizacionais, verificaram que a presença de uma cultura que preza por um ambiente pautado em boas relações interpessoais teve repercussões na confiança organizacional.

O estudo de David (2005) revelou que Relações Interpessoais e Segurança (fatores de QVT) foram preditores de comprometimento afetivo e normativo, respectivamente. Considerando o vínculo representado pelo comprometimento, é possível tecer uma comparação com a confiança depositada pelo empregado em sua organização, sendo que a QVT parece influenciar positivamente na criação desse vínculo.

A relação entre QVT e confiança organizacional também foi encontrada no estudo de Van der Berg e Martins (2013), que mostrou que as práticas gerenciais consideradas positivas pelos empregados e a experiência de QVT aumentam na mesma proporção que a relação de confiança entre o empregado e a organização. Sendo assim, é possível que, ao investir e estimular o trabalho em equipe, os espaços de recreação e atividades sociais, (David, 2005) as organizações estejam contribuindo para melhora dos vínculos e das relações interpessoais, e consequentemente, do padrão de confiança do empregado.

Os preditores de confiança na competência organizacional identificados na segunda análise de regressão foram os valores organizacionais Realização $(\beta=0,18, t=2,0, p<0,046)$ e Prestígio $(\beta=0,55, t=9,17$, $\mathrm{p}<0,001)$ e fatores de QVT Segurança $(\beta=0,19, \mathrm{t}=2,32$, $\mathrm{p}<0,021)$ e Saúde Física $(\beta=-0,19, \mathrm{t}=-3,38, \mathrm{p}<0,001)$. O modelo explicou $68 \%$ da variância $\left(R^{2}\right.$ ajustado $=0,66$; $\mathrm{F}(12,168)=30,303 ; \mathrm{p}<0,001)$. A percepção dos valores Realização e Prestígio indicam que os empregados tendem a confiar na competência da organização que é reconhecida e admirada pela sociedade em função de produtos e serviços ofertados.

O fator de QVT Segurança é outro preditor significativo de confiança na competência organizacional. Assim como no estudo de Batista (2010), esse resultado mostra que, se a organização é vista como capaz de ofertar salários, benefícios e garantias ao trabalhador, há maior tendência em ser percebida como confiável e estável financeiramente, proporcionando segurança em relação ao trabalho e ao futuro da organização. 
Psicologia: Ciência e Profissão Jan/Mar. 2017 v. 37 n¹, 7-17.

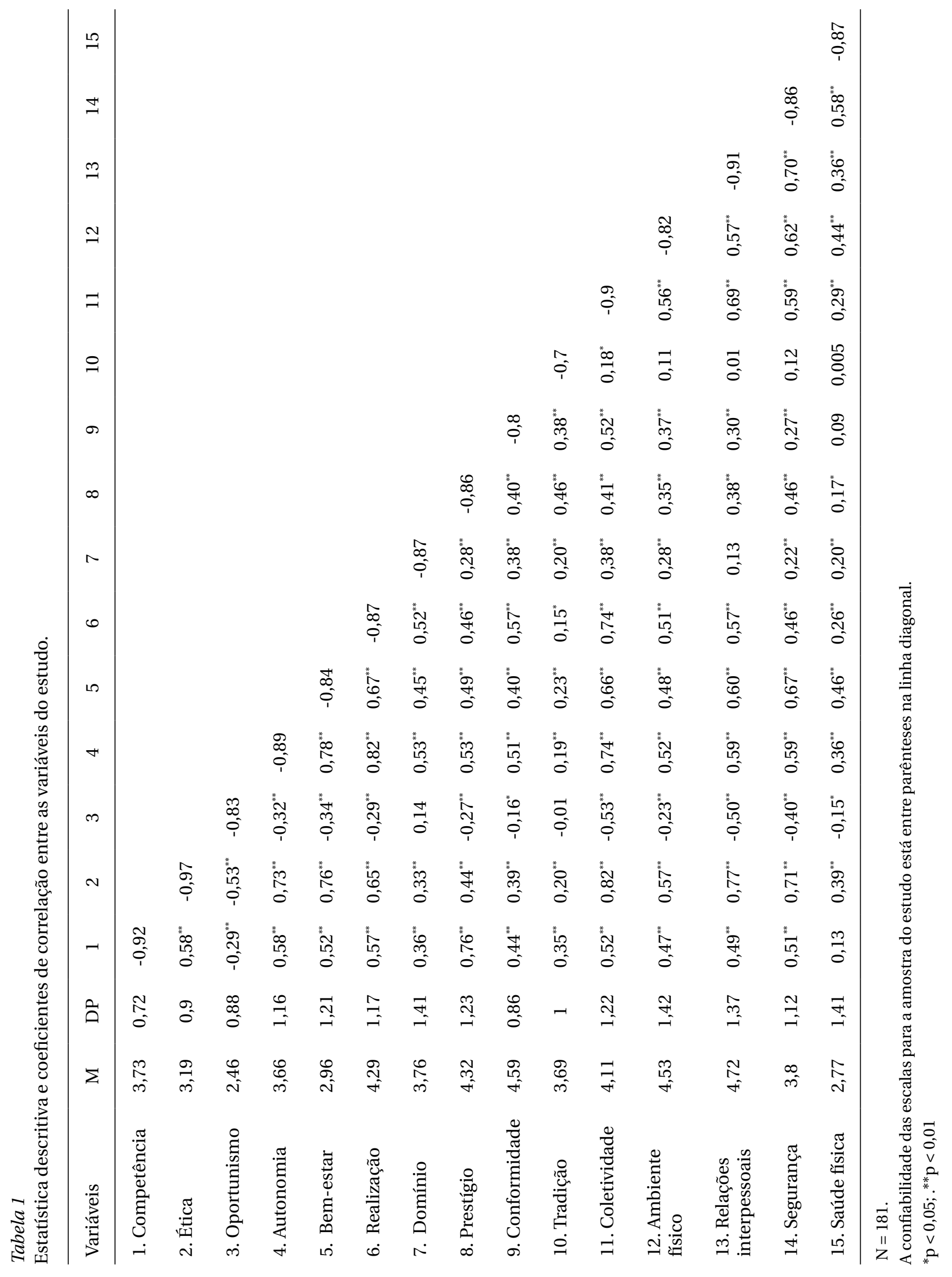


Tabela 2

Resumo das análises de regressão múltipla para as variáveis critério.

\begin{tabular}{|c|c|c|c|c|c|c|c|}
\hline \multirow{2}{*}{ Variáveis (critério) } & \multirow{2}{*}{ Preditores } & \multirow{2}{*}{ B } & \multirow{2}{*}{$\beta$} & \multirow{2}{*}{$\mathrm{p}$} & $\mathrm{R}$ & $\mathrm{R}^{2}$ & $\mathrm{R}^{2}$ \\
\hline & & & & & múltiplo & modelo & ajustado \\
\hline \multirow{4}{*}{$\begin{array}{l}\text { Componentes } \\
\text { éticos }\end{array}$} & VO Bem-estar & 0,19 & 0,26 & 0,001 & 0,91 & 0,82 & 0,81 \\
\hline & VO Coletividade & 0,32 & 0,43 & 0,001 & & & \\
\hline & $\begin{array}{l}\text { QVT Relações } \\
\text { interpessoais }\end{array}$ & 0,16 & 0,25 & 0,001 & & & \\
\hline & QVT Segurança & 0,11 & 0,14 & 0,028 & & & \\
\hline \multirow{4}{*}{$\begin{array}{l}\text { Competência } \\
\text { organizacional }\end{array}$} & VO Realização & 0,11 & 0,18 & 0,046 & 0,83 & 0,68 & 0,66 \\
\hline & VO Prestígio & 0,32 & 0,55 & 0,001 & & & \\
\hline & QVT Segurança & 0,12 & 0,19 & 0,021 & & & \\
\hline & QVT Saúde Física & $-0,1$ & $-0,19$ & 0,001 & & & \\
\hline \multirow{3}{*}{ Oportunismo } & VO Domínio & 0,22 & 0,34 & 0,001 & 0,68 & 0,47 & 0,43 \\
\hline & VO Prestígio & $-0,12$ & $-0,17$ & 0,031 & & & \\
\hline & VO Coletividade & $-0,45$ & $-0,62$ & 0,001 & & & \\
\hline
\end{tabular}

$\mathrm{VO}=$ valores organizacionais; $\mathrm{QVT}=$ qualidade de vida no trabalho.

O último preditor dessa variável critério é o fator de QVT Saúde Física. Contudo, o sentido da relação entre ambas pareceu incoerente, uma vez que uma organização que se propõe a realizar ações destinadas à saúde do trabalhador, como práticas de atividades físicas no local de trabalho, provavelmente não seria percebida como menos competente.

A competência organizacional refere-se à organização que possui conhecimento, domina bem as tecnologias em sua área de atuação; oferece produtos e serviços de qualidade aos seus clientes e apresenta estabilidade financeira que se manifesta na expectativa de continuidade e de futuro próspero. Verificou-se então, que a correlação bivariada entre Confiança na Competência Organizacional e Saúde física (QVT) é baixa e não significativa. Entretanto, na regressão observou-se que esse preditor foi significativo e apresentou valor de sinal oposto ao da correlação com a variável critério, sugerindo tratar-se de supressão: "um sinal de relações complexas entre variáveis preditoras na explicação da variável critério” (Abbad, \& Torres, 2002; Tabachnick, \& Fidell, 2001, p. 22).

Considerando esses indicativos, a variável QVT Saúde Física foi eliminada do modelo e uma nova análise de regressão com a exclusão de Saúde Física resultou em um modelo que explicou $66 \%$ da variância $\left(\mathrm{R}^{2}\right.$ ajustado $=0,64 ; \mathrm{F}(11,169)=30,16$; $\mathrm{p}<0,001$ ), permanecendo como preditores significativos somente os valores Prestígio $(\beta=0,57, t=9,431$, $\mathrm{p}<0,001)$ e Realização $(\beta=0,19, \mathrm{t}=2,086, \mathrm{p}<0,038)$.
Em se tratando de oportunismo, os preditores significativos foram somente nos valores organizacionais Domínio ( $\beta=0,34, \mathrm{t}=4,72, \mathrm{p}<0,001)$, Prestígio $(\beta=-0,17, \mathrm{t}=-2,17, \mathrm{p}<0,031)$ e Coletividade $(\beta=-0,62$, $\mathrm{t}=-5,95, \mathrm{p}<0,001)$. O modelo explicou $47 \%$ da variância $\left(\mathrm{R}^{2}\right.$ ajustado $\left.=0,43 ; \mathrm{F}(12,168)=12,384 ; \mathrm{p}<0,001\right)$. No caso desta variável critério, somente os valores ofereceram contribuições significativas. A busca de controle sobre pessoas e recursos assim como a busca de domínio do mercado poderia gerar ações organizacionais no sentido de obter vantagens sobre empregados e clientes, quando a oportunidade se apresentasse. Por outro lado, a percepção do valor Prestígio, representada pela busca de admiração e respeito da sociedade e a conduta ética da organização não estimularia ações oportunistas da organização.

Os resultados deste estudo e a literatura indicam que o alcance de um padrão de confiança dos empregados em relação às organizações necessita da presença de um conjunto complexo de fatores, já que cada aspecto da confiança possui preditores específicos.

\section{Conclusões}

Este estudo teve por objetivo principal verificar o impacto das variáveis QVT e percepção de valores organizacionais sobre a confiança do empregado na organização.

Os resultados referentes aos valores organizacionais traçam um cenário em que os empregados perce- 
bem consideravelmente o empenho das organizações em estabelecer limites, regras e modelos de comportamento (valor Conformidade), mas nem tanto em favorecer o Bem-Estar do Empregado. De maneira semelhante, em relação à QVT, os empregados referem satisfação com as Relações Interpessoais proporcionadas, mas pouca satisfação com Saúde Física. Esses dados suscitam um questionamento sobre a atenção dirigida pelas organizações às necessidades do empregado, enquanto ser complexo e enquanto recurso essencial para o funcionamento organizacional. Diante disso, aventa-se a possibilidade de estudos que busquem compreender a importância que as organizações dispensam à saúde física dos empregados, pensando-os como corpos que compõem o ambiente organizacional e, por isso, conforme Siqueira e Kurcgant (2012), precisam estar saudáveis e satisfeitos para continuar atendendo as próprias necessidades e as do mercado de trabalho.

A confiança do empregado na organização e a QVT também apresentaram correlações significativas, especialmente no que se refere às relações interpessoais, sinalizando para a importância de se manterem relações satisfatórias entre chefes e colegas de trabalho, evitando, por exemplo, o reconhecimento de competências individuais em detrimento das coletivas, por instigar a competitividade entre colegas e favorecer ambientes e relações hostis. A forma como acontecem as relações também está relacionada às normas de conduta definidas pela organização em seus valores, já que estes orientam as interações dos indivíduos entre si e com o meio externo, na forma de pensar, agir e tomar decisões (Deal, \& Kennedy, 1982; Pinto Neto, 2011; Pires, 2004). Assim, é possível notar uma interface entre aspectos relacionados à QVT, aos valores organizacionais e ao estabelecimento do vínculo de confiança.

As análises de regressão demonstraram que a hipótese de predição levantada neste estudo se confirma. Os valores organizacionais Bem-estar do Empregado, Coletividade e os fatores de QVT Relações interpessoais e Segurança tiveram um valor preditivo em relação à confiança nos componentes éticos. Desta forma, a percepção do empregado sobre a atenção da organização às suas necessidades, valorização dos aspectos coletivos, das relações entre empregados e clientes e o oferecimento de garantias de estabilidade aumentam suas chances de confiar na organização como sendo honesta e respeitosa.

A confiança na competência organizacional teve sua variância explicada pelos valores Realização e Prestígio, tendo sido os fatores de QVT Segurança e Saúde
Física eliminados devido à hipótese de supressão atribuída ao último fator. Organizações que têm como foco sua própria competência e a de seus colaboradores podem estimular uma relação de confiança com estes, que passam a percebê-las como organizações estáveis, seguras e com um futuro promissor. Este resultado deve ser visto com cautela em função da supressão, pois não é possível desconsiderar o alerta de Tabachnick e Fidell (2001) ao apontarem que um coeficiente de regressão grande para uma variável independente com baixa correlação com a variável critério pode ser enganoso em virtude desse fenômeno. Ou seja, a variável supressora é capaz de melhorar os efeitos de outra variável no conjunto das variáveis independentes, o que faz sugerir que diferentes estudos poderiam verificar se este fenômeno se repete em outras amostras.

A variância explicada para Oportunismo foi atribuída somente aos valores organizacionais Domínio, Prestígio e Coletividade, já que QVT não entrou na explicação desse fator de confiança. Nesse caso, entende-se que a busca por admiração e respeito da sociedade (Prestígio) não estimularia ações oportunistas sobre empregados e clientes e, por outro lado, tais ações poderiam ser estimuladas a partir do empenho organizacional em exercer controle sobre pessoas e recursos, assim como pela busca de domínio do mercado. Considerando Batista (2010) e Khetavath (2013), é possível sugerir que essas variáveis poderiam atuar como critério, ao supor que, quando a organização pauta suas práticas de forma a não prejudicar os clientes ou empregados, favorece a vivência de sentimentos de prazer no trabalho e possibilita uma visão de que a organização se atenta para a QVT.

O estudo sinaliza para a importância de conhecer e investir em variáveis que antecedam a confiança do empregado na organização, já que esse é um fator que propicia melhora da interação empregado-organização e que tem potencial para influenciar diversos outras instâncias no contexto organizacional, por exemplo, desempenho e produtividade. Apesar de não esgotar o assunto, os resultados obtidos podem beneficiar a construção do conhecimento que aborda a relação entre QVT, percepção dos valores organizacionais e confiança do empregado na organização, atentando para as lacunas que ainda existem em sua compreensão. Todavia, sugere-se que outros estudos sejam realizados a fim de se verificar como as variáveis aqui empregadas se comportam em outras amostras, testando suas potencialidades como explicativas, mediadoras e ou moderadoras nessa relação. 
Silva, U.L.; Oliveira, Á.F. (2016). Fatores influenciadores da confiança do empregado.

\section{Referências}

Abbad, G., \& Torres, C. V. (2002). Regressão múltipla stepwise e hierárquica em Psicologia Organizacional: aplicações, problemas e soluções. Estudos de Psicologia, 7(num esp), 19-29. https://doi.org/10.1590/S1413-294X2002000300004

An, J.Y., Yom, Y. H., \& Ruggiero, J. S. (2011). Organizational culture, quality of work life, and organizational effectiveness in Korean university hospitals. Journal of Transcultural Nursing, 22(1), 22-30. https://doi.org/10.1177/1043659609360849

Batista, A. A. S. (2010). Análise da qualidade de vida no trabalho utilizando um modelo de regressão logística (Dissertação de mestrado). Universidade Tecnológica Federal do Paraná, Ponta Grossa, PR.

Batista, R. L., \& Oliveira, A. F. (2012). Antecedentes da confiança do empregado na organização. Estudos de Psicologia, 17(2), 247-254. Recuperado de http://www.scielo.br/pdf/epsic/v17n2/08.pdf

Bigliardi, B., Dormio, A. I., Galati, F., \& Schiuma, G. (2012). The impact of organizational culture on the job satisfaction of knowledge workers. VINE Journal of Information and Knowledge Management Systems, 42(1), 36-51. https://doi.org/10.1108/03055721211207752

Buss, P. M., \& Pellegrini Filho, A. (2007). A saúde e seus determinantes sociais. Physis: Revista de Saúde Coletiva, 17(1), 77-93. https://doi.org/10.1590/S0103-73312007000100006

Dancey, C. P., \& Reidy, J. (2006). Estatística sem matemática para psicologia. Porto Alegre, RS: Artmed.

David, L. M. L. (2005). Qualidade de vida no trabalho e comprometimento organizacional: análise de suas relações em uma unidade do exército (Dissertação de mestrado). Universidade de Brasília, Brasília, DF.

Deal, T. E., \& Kennedy, A. A. (1982). Corporate cultures the rites and rituals of corporate life. Londres: Penguin.

DeConinck, J. B. (2010). The effect of organizational justice, perceived organizational support, and perceived supervisor support on marketing employees' level of trust. Journal of Business Research, 63(12), 1349-1355. https://doi.org/10.1016/j.jbusres.2010.01.003

Estivalete, V. F. B., \& Andrade, T. (2012). A influência dos valores organizacionais na percepção de suporte organizacional com base na concepção dos colaboradores do setor bancário. RAM. Revista de Administração Mackenzie, 13(3), 214-244. https://doi.org/10.1590/S1678-69712012000300010

Estivalete, V. F. B., Andrade, T., Gomes, T. C., \& Costa, V. F. (2012). Valores organizacionais no Brasil: uma análise da produção científica na área da administração nos últimos 10 anos. Gestão Contemporânea, 9(12), 43-68. Recuperado de http://seer4.fapa.com.br/index.php/arquivo/article/view/150/108

Fard, A. M., Zahed-Babelan, A., \& Sattari, S. (2013). The relation between organizational culture and organizational trust in customs headquarters of Iran. International Research Journal of Applied and Basic Sciences, 4(1), 164-167. Recuperado de http://www.irjabs.com/files_site/paperlist/r_627_130116141018.pdf

Field, A. (2009). Descobrindo a estatística usando SPSS. Porto Alegre, RS: Artmed.

Gorenak, M., \& Košir, S. (2012). The importance of organizational values for organization. In Management, Knowledge and Learning International Conference PP. 563-569), Slovenia. Recuperado em 15 de outubro de 2013, de http://www.issbs.si/press/ISBN/978-961-6813-10-5/papers/ML12_117.pdf

Gorzoni, P. M. (2010). Cultura organizacional e qualidade de vida no trabalho: um estudo com funcionários de um restaurante fast-food (Dissertação de mestrado). Universidade de São Paulo, São Paulo, SP.

Guardani, F., Teixeira, M. L. M., Bido, D. S., \& Mazzon, J.A. (2013). A relação entre valores, práticas organizacionais e confiança de clientes no setor de serviços. Produção, 23(4), 806-817. Recuperado de http://www.scielo.br/scielo. php?script=sci_arttext\&pid=S0103-65132013000400011

Hackman, J. R., \& Oldham, G. R. (1975). Development of the job diagnostic survey. Journal of Applied Psychology, 60(2), 159-170. Recuperado de http://www.jwalkonline.org/upload/pdf/Hackman\%20\%26\%20Oldham\%20 (1975)\%20-\%20Development\%20of\%20the\%20JDS.pdf

Hair, J. F., Anderson, R. E., Tatham, R. L., \& Black, W. C. (2005). Análise multivariada de dados (5a ed.; S. S. Adonai, \& C. N. Anselmo, Trads.). Porto Alegre, RS: Bookman.

Ianaguivara, C. M. G. A. (2011). Confiança da empregado na organização: revalidação de instrumento de medida (Dissertação de mestrado). Universidade Federal de Uberlândia, Uberlândia, MG. 
Khetavath, P. S. (2013). A comparative study of quality of work life in public and private sector organizations. In Tenth AIMS International Conference on Management (pp. 2960-2966), Hyderabad, India,.

Kramer, R. M. (1999). Trust and distrust in organizations: emerging perspectives, enduring questions. Annual Review of Psychology, 50(1), 569-598. https:// doi.org/10.1146/annurev.psych.50.1.569

Medeiros, J. P. (2007). Qualidade de vida no trabalho da EMATER-RN: validação de um instrumento síntese de pesquisa e diagnóstico (Dissertação de mestrado). Universidade Federal do Rio Grande do Norte, Natal, RN.

Medeiros, L. F. R., \& Ferreira, M. C. (2011). Qualidade de vida no trabalho: uma revisão da produção científica de 1995-2009. Gestão Contemporânea, 8(9), 9-34. Recuperado de http://www.ergopublic.com.br/arquivos/1359565902.29-arquivo.pdf

Miles, J., \& Schevlin, M. (2001). Applying regression \& correlation: a guide for students e researchers. London: Sage.

Oliveira, A. F. (2004). Confiança do empregado na organização: impacto dos valores pessoais, organizacionais e da justiça organizacional (Tese de doutorado). Instituto de Psicologia Universidade de Brasília, Brasília, DF.

Oliveira, A. F., \& Souza, M. A. (2014). Confiança do empregado na organização: o impacto dos valores pessoais e organizacionais. Revista Psicologia: Organizações e Trabalho, 14(2), 204-217. Recuperado de http://pepsic.bvsalud.org/scielo.php?script=sci_arttext\&pid=S1984-66572014000200007

Oliveira, A. F., \& Tamayo, A. (2008). Confiança do empregado na organização. In M. M. M. Siqueira (Ed.), Medidas do comportamento organizacional: ferramentas de diagnóstico e de gestão (pp. 97-109). Porto Alegre, RS: Artmed.

Oliveira, A. F., \&Tamayo, A. (2004). Inventário de perfis de valores organizacionais. Revista de Administração, 39(2),129-140.

Özyilmaz, Y. D. A. (2010). Vertical trust in organizations: a review of empirical studies over the last decade. Journal of SocialSciencesInstitute, 7(13), 1-28. Recuperadodehttp://sbed.mku.edu.tr/article/view/1038000588/1038000380

Paula, R. A. (2009). A confiança e suas interfaces com os valores organizacionais em microempresas (Dissertação de mestrado). Faculdades de Pedro Leopoldo, Pedro Leopoldo, MG.

Pinto Neto, B. (2011). Valores organizacionais presentes nos órgãos administrativos de uma instituição de ensino superior federal de Minas Gerais: um estudo de caso (Dissertação de mestrado). Faculdades de Pedro Leopoldo, Pedro Leopoldo, MG.

Pires, J. C. S. (2004). Relações entre valores individuais, valores organizacionais e programa de qualidade de vida no trabalho (Dissertação de mestrado). Universidade Católica de Goiás, Goiânia, GO.

Purohit, B., Patel, D., \& Purohit, S. (2014). A study of organizational values in government run primary health centres in India. Journal of Health Management, 16(2), 303-313. https://doi.org/10.1177/0972063414526119

Rousseau, D. M., Sitkin, S. B., Burt, R. S., \& Camerer, C. (1998). Not so different after all: a cross discipline view of trust. Academy of Management Review, 23(3), 393-404. https://doi.org/10.5465/AMR.1998.926617

Silva, N., \& Tolfo, S. R. (1999). Qualidade de vida no trabalho e cultura organizacional: um estudo no ramo hoteleiro de Florianópolis. Convergência, 20(6), 275-300. Recuperado de http://convergencia.uaemex.mx/article/view/1877/1429

Silva, N., \& Zanelli, J. C. (2004). Cultura organizacional. In J. C. Zanelli, J. E. Borges- Andrade, \& A. V. B. Bastos (Eds.), Psicologia, organizações e trabalho no Brasil. (pp. 407-442). Porto Alegre, RS: Artmed.

Silva, N., Zanelli, J. C., \& Tolfo, S. R. (2014). Cultura organizacional. In J. C. Zanelli, J. E. Borges-Andrade, \& A. V. B. Bastos (Eds.), Psicologia, organizações e trabalho no Brasil. (pp. 491-525). Porto Alegre, RS: Artmed.

Sinha, C. (2012). Factors affecting quality of work life: empirical evidence from indian organizations. Australian Journal of Business and Management Research, 1(11), 31-40.

Siqueira, V. T. A., \& Kurcgant, P. (2012). Satisfação no trabalho: indicador de qualidade no gerenciamento de recursos humanos em enfermagem. Revista da Escola de Enfermagem da USP, 46(1), 151-157. https://doi.org/10.1590/S0080-62342012000100021

Tabachnick, B. G., \& Fidell, L. S. (2001). Using multivariate statistics. New York, NY: Allyn and Bacon.

Tamayo, A. (1998). Valores organizacionais: sua relação com satisfação no trabalho, cidadania organizacional e comprometimento afetivo. Revista de Administração, 33(3), 56-63.

Tolfo, S. R., \& Piccinini, V. C. (2011). Qualidade de vida no trabalho nas melhores empresas para se trabalhar no Brasil: descompassos entre teoria e prática. In A. S. Sant’Anna, \& Z. M. Kilimnik (Orgs.), Qualidade de vida no trabalho: fundamentos e abordagens (pp. 85-112). Rio de Janeiro, RJ: Elsevier. 
Tolfo, S. R., Silva, N., \& Luna, I. N. (2009). Cultura organizacional, identidade e qualidade de vida no trabalho: articulações e sugestões de pesquisas em organizações. Pesquisas e Práticas Psicossociais, 4(1), 6-16. Recuperado de http://www.ufsj.edu.br/portal-repositorio/File/revistalapip/volume4_n1/tolfo_e_outros.pdf

Tzafrir, S. S., \& Dolan, S. L. (2004). Trust me: a scale for measuring manager-employee trust. Management Research, 2(2), 115-132. https://doi.org/10.1108/15365430480000505

Valizadeh, A., \& Ghahremani, J. (2012). The relationship between organizational culture and quality of working life of employees. European Journal of Experimental Biology, 2(5), 1722-1727. Recuperado de http://www.imedpub. $\mathrm{com} /$ articles/the-relationship-between-organizational-culture-and-quality-of-working-life-of-employees.pdf

Van der Berg, Y. (2011). The relationship between organisational trust and quality of work life (Master thesis). University of South Africa, Africa

Van der Berg, Y., \& Martins, N. (2013). The relationship between organisational trust and quality of work life. $S A$ Journal of Human Resource Management, 11(1), 1-13. https:// doi.org/10.4102/sajhrm.v1 li1.392

Watson, M. L. (2005). Can there be just one trust? A cross-disciplinary identification of trust definitions and measurement, The Institute for Public Relations, 1-25. Recuperado de http://www.instituteforpr.org/wp-content/uploads/Cross-Disciplinary-Identification-of-Trust.pdf

Uanisléia Lima da Silva

Mestre em Psicologia pela Universidade Federal de Uberlândia, Uberlândia - MG. Brasil.

E-mail: uanisleia@yahoo.com.br

Áurea de Fátima Oliveira

Doutora em Psicologia pela Universidade de Brasília, Brasília - DF. Docente do Programa de Pós-Graduação em Psicologia da Universidade Federal de Uberlândia, Uberlândia - MG. Brasil.

E-mail: oliveira.aureaf@yahoo.com.br

Endereço para envio de correspondência:

Universidade Federal de Uberlândia

Av. Mato Grosso, 3424 - Umuarama. CEP: 38405-314

Uberlândia, MG, Brasil

Recebido 02/01/2015

Aprovado 14/12/2016

Received 01/02/2015

Approved 12/14/2016

Recibido 02/01/2015

Aceptado 14/12/2016

Como citar: Silva, U. L., \& Oliveira, A. F. (2017). Qualidade de vida e valores nas organizações: impactos na confiança do empregado. Psicologia: Ciência e Profissão, 37(1): 7-17. https://doi.org/10.1590/1982-3703000012015

How to cite: Silva, U. L., \& Oliveira, A. F. (2017). Quality of life and values in organizations: impacts on the employee trust. Psicologia: Ciência e Profissão, 37(1): 7-17. https://doi.org/10.1590/1982-3703000012015

Cómo citar: Silva, U. L., \& Oliveira, A. F. (2017). Calidad de vida y valores en las organizaciones: impactos en la confianza del empleado. Psicologia: Ciência e Profissão, 37(1): 7-17. https://doi.org/10.1590/1982-3703000012015 